\title{
Higher Order Strongly Biconvex Functions and Biequilibrium Problems
}

\author{
Muhammad Aslam Noor*, Khalida Inayat Noor \\ Department of Mathematics, COMSATS University Islamabad, Islamabad, Pakistan \\ Email: *noormaslam@gmail.com, khalidan@gmail.com
}

How to cite this paper: Noor, M.A. and Noor, K.I. (2021) Higher Order Strongly Biconvex Functions and Biequilibrium Problems. Advances in Linear Algebra \& Matrix Theory, 11, 31-53.

https://doi.org/10.4236/alamt.2021.112004

Received: March 12, 2021

Accepted: June 1, 2021

Published: June 4, 2021

Copyright $\odot 2021$ by author(s) and Scientific Research Publishing Inc. This work is licensed under the Creative Commons Attribution International License (CC BY 4.0).

http://creativecommons.org/licenses/by/4.0/

(c) (i) Open Access

\begin{abstract}
In this paper, we introduce and study some new classes of biconvex functions with respect to an arbitrary function and a bifunction, which are called the higher order strongly biconvex functions. These functions are nonconvex functions and include the biconvex function, convex functions, and $k$-convex as special cases. We study some properties of the higher order strongly biconvex functions. Several parallelogram laws for inner product spaces are obtained as novel applications of the higher order strongly biconvex affine functions. It is shown that the minimum of generalized biconvex functions on the $k$-biconvex sets can be characterized by a class of equilibrium problems, which is called the higher order strongly biequilibrium problems. Using the auxiliary technique involving the Bregman functions, several new inertial type methods for solving the higher order strongly biequilibrium problem are suggested and investigated. Convergence analysis of the proposed methods is considered under suitable conditions. Several important special cases are obtained as novel applications of the derived results. Some open problems are also suggested for future research.
\end{abstract}

\section{Keywords}

Biconvex Functions, Convex Functions, $k \beta$-Convex Functions, $k \beta$-Convex Sets, Parallelogram Laws, Biequilibrium Problems, Bivariational Inequalities, Iterative Methods, Convergence Analysis

\section{Introduction}

Variational inequality theory, which was introduced and studied by Stampacchia [1] can be viewed as an important and significant extension of the variational principles, the origin of which can be traced back to Euler, Lagrange, Newton, and Bernoulli brothers. This theory has had a great impact and influence in the 
development of several branches of pure, applied, and engineering sciences. It has been shown that variational inequalities include complementarity problems, fixed-point, optimization problems, and game theory as special cases. Variational inequalities have drawn much attention from researchers because of their importance as a core model for studying many mathematical problems which include convex programming, equilibrium problem, inclusion problem, split feasibility problem, complementarity problem, minimization problem, see [2] [3].

It is well known that the minimum of differentiable convex function on a convex set can be characterized by an inequality, which is called the variational inequality. This simple fact has influenced several branches of pure and applied sciences. For the formulation, applications, numerical methods, and other aspects of the variational inequalities, see [4]-[17] and the references therein. Variational inequalities can be viewed as a novel and significant extension of the variational principles. Blum and Oettlie [18] and Noor and Oettlie [19] introduced the concept of equilibrium, which has appeared as an interesting generalization of the variational inequalities. Equilibrium problems include variational inequalities and fixed point problems as special cases. Several equilibrium problems provide us a unified framework to study unrelated arising in transportation, economics, and management sciences in a unified framework.

In recent years, several extensions and generalizations of the convex sets and convex functions have been considered and investigated. Noor et al. [20] [21] introduced the concept of biconvex sets and biconvex functions. It is known that the differentiable biconvex functions are biconvex functions. The converse also holds under certain conditions, see [20]. Noor et al. [20] [21] proved that the minimum of the differentiable biconvex functions on the biconvex set can be characterized by a class of variational inequalities, which is known as the bivariational inequality. Noor [20] [21] studied the biconvex equilibrium problems, which can be viewed as an interesting and significant extension of the variational inequalities. For the recent developments in bivariational inequalities and biconvex equilibrium problems, see [20] [21] [22] and the references therein. These results have inspired a great deal of subsequent work, which has expanded the role and applications of convexity in nonlinear optimization and engineering sciences.

In many problems, a set may not be a convex set. To overcome this drawback, the underlying set can be made a $k$-convex set with respect to an arbitrary function. Micherda et al. [23], Hazy [24], and Crestescu et al. [25] defined the socalled $(h, k)$ convex function which is a natural generalization of the usual convexity, the $s$-convexity in the first and second sense. It is worth mentioning that $k(t)=t \mathrm{e}^{i \varphi}$, the $\varphi$-convex functions were introduced and studied by Noor [26].

We would like to point out that biconvex sets, biconvex functions, $k$-convex sets, and $k$-convex functions are different generalizations of convex sets and convex functions in various directions. These types of functions have played a 
leading role in the developments of various branches of pure and applied sciences. It is natural to unify these classes of functions and to investigate their characterizations. Motivated and inspired by the recent activities in these areas, we introduce some new classes of biconvex sets and biconvex functions involving an arbitrary function $k$, which are called modified $k$-biconvex sets and $k$-biconvex functions. These new classes of $k$-biconvex sets and $k$-biconvex functions include the $\varphi$-biconvex sets, $\varphi$-biconvex and Toader type $k$-convex sets and $k$-convex functions. These $k$-biconvex functions can be viewed as modified refinement of the $(h, k)$ convex functions of Micherda et al. [23] and Hazy [24]. Several new concepts are defined and their properties have been studied. We prove that the minimum of the differential $k$-biconvex functions on the $k$ biconvex sets can be characterized by a class of equilibrium problems. This result inspired us to consider the biequilibrium problems.

As a result of interaction between different branches of mathematical and engineering sciences, we now have a variety of techniques to suggest and analyze various iterative algorithms for solving equilibrium problems, variational inequalities and related optimization problems. Analysis of these problems requires a blend of techniques and ideas from convex analysis, functional analysis, numerical analysis and nonsmooth analysis. There are several methods for solving variational inequalities and equilibrium problems. Due to the nature of the biequilibrium problems, projection and resolvent methods can not be applied for solving directional equilibrium-like problems. To overcome these difficulties, one usually uses the auxiliary principle technique, which is mainly due to Glowinski et al. [4]. The main idea involving this technique is to first consider an auxiliary problem and then to show that the solution of the auxiliary problem is the solution of the original problem by using the fixed-point approach. Noor [9] [11] [12] [19] and Noor et al. [13] [14] [15] [16] have used this approach to suggest and analyze some iterative methods for solving various classes of variational inequalities and equilibrium problems. It is worth mentioning that one can use several principles to suggest auxiliary problem associated with the original problem. Bregman [27] introduced the convex functions involving the distance functions and discussed its applications in convex programming and optimization problems. Noor et al. [13] [14] [15] [16] and Zhu and Marcote [17] used the auxiliary principle involving the Bregman functions. They have suggested and analyzed a wide class of iterative method for solving variational inequalities and equilibrium problems. They have shown [2] [3] that the Bregman functions have some practical important types of functions such as Burg entropy, which is very important in information theory and Shannon entropy, having applications in several areas of applied mathematics such as machine learning. This shows that the entropy methods have been used in the development of methods for solving equilibrium problems in various ways. Thus one concluded the entropy technique has been used effectively in studying the variational inequalities and equilibrium problems. We again show that the auxiliary principle technique involv- 
ing Bregman functions can be used to suggest some iterative schemes for solving the higher order biequilibrium problems. We prove that the convergence of these methods requires either pseudomonotonicity or partially relaxed strongly monotonicity. These are weaker conditions than monotonicity. As special cases, we obtain iterative schemes for solving biequilibrium problems and related optimization problems. The comparison of these methods with other methods is a subject of future

In section 2, we introduce and study some new concepts regarding higher order strongly biconvex functions and their properties are discussed. Main properties of the higher order strongly biconvex are discussed in Section 3. Various parallelograms have been derived in Section 4 as applications of higher order strongly $k$-biconvex affine functions, which can be used to characterize several inner product and Banach spaces. In Section 5, we show that the optimality conditions of the higher order strongly generalize biconvex functions can be characterized by biequilibrium problems. Using the auxiliary technique coupled with Bregman functions, we have suggested and investigated several iterative methods for solving the higher order strongly biequilibrium problems. Convergence of the proposed methods is considered using the pseudomonotone operators, which is weaker condition than monotonicity. Several special cases are discussed as applications of our results. Comparison with other methods and implementation of the suggested methods need further efforts. Several open problems are discussed for future research.

\section{Preliminaries}

Let $K_{k}$ be a nonempty closed set in a normed space $H$. We denote by $\langle\cdot, \cdot\rangle$ and $\|\cdot\|$ the inner product and norm, respectively.

Definition 2.1. The set $K_{k \beta}$ is said to be k-biconvex set with respect to arbitrary function $k$ and the bifunction $\beta(.-$.), if

$$
u+k(\lambda) \beta(v-u) \in K_{k \beta}, \quad \forall u, v \in K_{k \beta}, \quad \lambda \in[0,1] .
$$

Clearly, for $k(\lambda)=\lambda$, the set $K_{k \beta}$ is a biconvex set $K_{\beta}$, which was introduced and studied by Noor and Noor [20].

Definition 2.2. [20] [21] The set $K$ is said to be biconvex set with respect to arbitrary the bifunction $\beta(.-$.$) , if$

$$
u+\lambda \beta(v-u) \in K_{\beta}, \quad \forall u, v \in K_{\beta}, \quad \lambda \in[0,1] .
$$

If $\beta(v, u)=v-u$, then (2.1) reduces to:

Definition 2.3. [23] [24] The set $K_{k}$ is said to be k-convex set with respect to arbitrary function $k$, if

$$
u+k(\lambda)(v-u) \in K_{k}, \quad \forall u, v \in K_{k}, \quad \lambda \in[0,1] .
$$

which was introduced by Hazy [13]. Also see [25] for relevant literature.

From now onwards, the set $K_{k \beta}$ is a $k$-biconvex set, unless otherwise specified. 
We now introduce the concept of higher order generalized biconvex function with respect to an arbitrary function $k$ and bifunction $\beta(.-$.).

Definition 2.4. The function $f$ on $K_{k \eta}$ is called higher order generalized biconvex function, if there exists an arbitrary function $k$, bifunction $\beta(.-$.$) and a$ constant $v$, such that

$$
\begin{aligned}
& f(u+k(\lambda) \beta(v-u)) \leq(1-k(\lambda)) f(u)+k(\lambda) f(v) \\
& -v\left\{(k(\lambda))^{p}(1-k(\lambda))+k(\lambda)(1-k(\lambda))^{p}\right\}\|\beta(v-u)\|^{p}, \\
& \forall u, v \in K_{k \beta}, \lambda \in[0,1] .
\end{aligned}
$$

If $k(\lambda)=\lambda$, then definition 2.4 reduces to:

Definition 2.5. The function $f$ on $K_{k \beta}$ is called higher order generalized biconvex function, if there exists an arbitrary function $k$, bifunction $\beta(.-$.$) and a$ constant $v$, such that

$$
\begin{aligned}
& f(u+\lambda \beta(v-u)) \leq(1-\lambda) f(u)+\lambda f(v) \\
& -v\left\{\lambda^{p}(1-\lambda)+\lambda(1-\lambda)^{p}\right\}\|\beta(v-u)\|^{p}, \\
& \forall u, v \in K_{k \beta}, \lambda \in[0,1] .
\end{aligned}
$$

The higher order biconvex functions were introduced and studied by Noor et al. [20] [21]. Obviously every higher order $k(\lambda)$ biconvex function with $k(\lambda)=\lambda$ is a higher order biconvex function, but the converse may is not true.

If $\beta(v, u)=v-u$, then higher order $k$-biconvex functions collapse to:

Definition 2.6. The function $f$ on $K_{k}$ is called higher order $k$-convex function, if there exists an arbitrary function $k$, and a constant $v$ such that

$$
\begin{aligned}
& f(u+k(\lambda)(v-u)) \leq(1-k(\lambda)) f(u)+k(t) f(v) \\
& -v\left\{(k(\lambda))^{p}(1-k(\lambda))+k(\lambda)(1-k(\lambda))^{p}\right\}\|v-u\|^{p}, \\
& \forall u, v \in K_{k}, \lambda \in[0,1] .
\end{aligned}
$$

If $k(\lambda)=\lambda, \beta(v, u)=v-u$, then definition 2.6 reduces to:

Definition 2.7. The function $f$ on $K$ is called higher order strongly convex function, if there exists a constant $v$, such that

$$
\begin{aligned}
& f(u+\lambda(v-u)) \leq(1-\lambda) f(u)+\lambda f(v) \\
& -v\left\{\lambda^{p}(1-\lambda)+\lambda(1-\lambda)^{p}\right\}\|v-u\|^{p}, \\
& \forall u, v \in K, \lambda \in[0,1], p>1 .
\end{aligned}
$$

Higher order convex functions and their variant forms were introduced and studied by Mohsin et al. [28] and Noor et al. [15] [22] recently. It is worth mentioning that these concepts were also represent significant refinement and improvement of the concepts of higher order functions considered in Alabdali et al. [29], Olbrys [30], Hazy [24], Lin et al. [6] and Mako et al. [31]. It has been shown [15] [28] that parallelogram laws for Banach spaces can be obtained as novel applications of the higher order convex affine functions, which have application in various fields of mathematical and engineering sciences including 
prediction theory.

For $\lambda=1$, the $k$-convex function reduces to:

Condition $\boldsymbol{A}$ The function $f$ is said to satisfy

$$
f(u+k(1) \beta(v-u)) \leq f(v), \quad \forall u, v \in K_{k \beta},
$$

which is called the condition $A$.

Definition 2.8. The function $f$ on $K_{k \beta}$ is said to be higher order strongly generalized quasi $k$-biconvex function, if there exist a function $k$ and a constant $v$ such that

$$
\begin{aligned}
& f(u+k(\lambda) \beta(v-u)) \leq \max \{f(u), f(v)\} \\
& -v\left\{(k(\lambda))^{p}(1-k(\lambda))+k(\lambda)(1-k(\lambda))^{p}\right\}\|\beta(v-u)\|^{p}, \\
& \forall u, v \in K_{k \beta}, \lambda \in[0,1], p>1 .
\end{aligned}
$$

Definition 2.9. The function $f$ on $K_{k \beta}$ is said to be higher order strongly generalized logarithmic biconvex function, if there exist a function $k$ and the bifunction $\eta(.-$.$) , such that$

$$
\begin{aligned}
& f(u+k(\lambda) \beta(v-u)) \leq(f(u))^{1-k(\lambda)}(f(v))^{k(\lambda)} \\
& -v\left\{(k(\lambda))^{p}(1-k(\lambda))+k(\lambda)(1-k(\lambda))^{p}\right\}\|\beta(v-u)\|^{p}, \\
& \forall u, v \in K_{k \beta}, \lambda \in[0,1], p>1 .
\end{aligned}
$$

where $f(\cdot)>0$.

We note that, if $\mu=0$, then definition 2.9 reduces to:

Definition 2.10. The function $f$ on $K_{k \beta}$ is said to be logarithmic biconvex function, if there exist a function $k$, such that

$$
f(u+k(\lambda) \beta(v-u)) \leq(f(u))^{1-k(\lambda)}(f(v))^{k(\lambda)}, \forall u, v \in K_{k \beta}, \lambda \in[0,1],
$$

or equivalently, it can be written as:

Definition 2.11 The function $f$ on $K_{k \beta}$ is said to be higher order strongly logarithmic generalized biconvex function, if there exist a function $k$, such that

$$
\begin{aligned}
& \log f(u+k(\lambda) \beta(v-u)) \\
& \leq(1-k(\lambda)) \log f(u)+k(\lambda) \log f(v), \forall u, v \in K_{k \beta}, \lambda \in[0,1],
\end{aligned}
$$

Using this idea, we can define the higher order logarithmic generalized biconvex functions as:

Definition 2.12. The function $f$ on $K_{k \beta}$ is said to be higher order strongly logarithmic generalized biconvex function, if there exist a function $k$, such that

$$
\begin{gathered}
\log f(u+k(\lambda) \beta(v-u)) \leq(1-k(\lambda)) \log f(u)+k(\lambda) \log f(v), \\
-v\left\{(k(\lambda))^{p}(1-k(\lambda))+k(\lambda)(1-k(\lambda))^{p}\right\}\|\beta(v-u)\|^{p} \\
\forall u, v \in K_{k \beta}, \lambda \in[0,1],
\end{gathered}
$$

which appears to be a new one.

From the above definitions, we have: 


$$
\begin{aligned}
& f(u+k(\lambda) \beta(v, u)) \\
\leq & (f(u))^{1-k(\lambda)}(f(v))^{k(\lambda)}-v\left\{(k(\lambda))^{p}(1-k(\lambda))+k(\lambda)(1-k(\lambda))^{p}\right\}\|\beta(v-u)\|^{p} \\
\leq & (1-k(\lambda)) f(u)+k(\lambda) f(v) \\
& -v\left\{(k(\lambda))^{p}(1-k(\lambda))+k(\lambda)(1-k(\lambda))^{p}\right\}\|\beta(v-u)\|^{p} \\
\leq & \max \{f(u), f(v)\}-v\left\{(k(\lambda))^{p}(1-k(\lambda))+k(\lambda)(1-k(\lambda))^{p}\right\}\|\beta(v-u)\|^{p}, \\
& \forall u, v \in K_{k \beta}, \lambda \in[0,1],
\end{aligned}
$$

This shows that higher order strongly logarithmic generalized biconvex function $\Rightarrow$ higher order strongly generalized biconvex functions and higher order strongly generalized biconvex functions $\Rightarrow$ higher order strongly generalized quasi biconvex functions, but the converse is not true.

We remark that, if $F$ is both higher order strongly biconvex function and higher order strongly biconcave function, then we can define the following new concept.

Definition 2.13. The function $f$ on $K_{k \beta}$ is called higher order strongly biconvex affine function, if there exist an arbitrary function $k$ and a constant $v$ such that

$$
\begin{aligned}
& f(u+k(\lambda) \beta(v-u))=(1-k(\lambda)) f(u)+k(\lambda) f(v) \\
& -v\left\{(k(\lambda))^{p}(1-k(\lambda))+k(\lambda)(1-k(\lambda))^{p}\right\}\|\beta(v-u)\|^{p}, \\
& \forall u, v \in K_{k \beta}, \lambda \in[0,1], p>1 .
\end{aligned}
$$

For proper and suitable choice of the functions $k(\lambda)$ and the bifunction $\beta(.-$.), one can a wide classes of higher order generalized biconvex functions and their variant forms, see [32] [33] [34] [35]. This shows that the concepts introduced in this paper are quite flexible and general ones.

We also need the following assumption regarding the bifunction $\eta(.-$.$) and$ the function $k(\lambda)$.

Condition M. Let $\beta(.-):. K_{k \beta} \times K_{k \beta} \rightarrow H$ satisfy assumptions:

1) $\beta(k(\lambda) \beta(v-u))=-k(\lambda) \beta(v-u)$

2) $\beta(v-u-k(\lambda) \beta(v-u))=(1-k(\lambda)) \beta(v-u), \forall u, v \in K_{k \beta}, \lambda \in[0,1]$.

Remark 2.1. Let

$$
\beta(v-u)=\beta(v-z)+\beta(z-u), \quad \forall u, v \in K_{k \beta} .
$$

Clearly $\beta(u-v)=0$, if and only if, $u=v, \forall u, v \in K_{k \beta}$, and

$$
\beta(v-u)+\beta(u-v)=0, \forall u, v \in k_{k \beta} .
$$

that is, the bifunction $\beta(,-$,$) is skew symmetric.$

We now introduce the concept of $k$-directional derivative, which is mainly due to Noor [26].

Definition 2.14. We define the k-directional derivative of $\mathrm{f}$ at a point $u \in K_{k \beta}$ 
in the direction $v \in K_{k \beta}$ by

$$
\begin{aligned}
D f(u, \beta(v-u)) & =f_{k \beta}^{\prime}(u, \beta(v-u)) \\
& =\lim _{\lambda \rightarrow 0^{+}}\left\{\frac{f(u+k(\lambda) \beta(v-u))-f(u)}{k(\lambda)}\right\} .
\end{aligned}
$$

Note that for $k(\lambda)=\lambda$ and $\beta(v-u)=v$, the $k$-directional derivative of $f$ at $u \in K$ in the direction $v \in K$ coincides with the usual directional derivative of $f$ at $u$ in a direction $v$ given by

$$
D f(u, v):=f^{\prime}(u, v)=\lim _{\lambda \rightarrow 0^{+}} \frac{f(u+t v)-f(u)}{\lambda} .
$$

It is well known that the function $v \rightarrow f_{k}^{\prime}(u, v-u)$ is subadditive, positively homogeneous.

Definition 2.15. A differentiable function $f$ on the biconvex set $K_{k \beta}$ is said to be higher order strongly pseudo $k$-biconvex function, iff, if there exists a constant $\mu>0$ such that

$$
f_{k \beta}^{\prime}(u, \beta(v-u))+\mu\|\beta(v-u)\|^{p} \geq 0 \Rightarrow f(v)-f(u) \geq 0, \quad \forall u, v \in K_{k \beta}, p \geq 1 .
$$

Definition 2.16. A differentiable function $f$ on $K_{k \beta}$ is said to be higher order strongly quasi- $k$-biconvex function, iff, if there exists a constant $\mu>0$ such that

$$
\begin{gathered}
f(v) \leq f(u) \\
\Rightarrow \\
f_{k \beta}^{\prime}(u, \beta(v-u))+\mu\|\beta(v-u)\|^{p} \leq 0, \quad \forall u, v \in K_{k \beta}, p \geq 1 .
\end{gathered}
$$

Definition 2.17. The function $f$ on the set $K_{k \beta}$ is said to be pseudo- $k \beta$ biconvex, iff,

$$
f_{k \beta}^{\prime}(u, \beta(v-u)) \geq 0 \Rightarrow f(v) \geq f(u), \quad \forall u, v \in K_{k \beta} .
$$

Definition 2.18. The differentiable function $f$ on the $K_{k \beta}$ is said to be quasi $k$-biconvex function, iff,

$$
f(v) \leq f(u) \Rightarrow f_{k \beta}^{\prime}(u, \beta(v-u)) \leq 0, \quad \forall u, v \in K_{k \beta} .
$$

I All these new concepts may play important and fundamental part in the developments of mathematical programming and optimization theory.

\section{Properties of Biconvex Functions}

In this section, we consider some basic properties of higher order strongly generalized biconvex functions on the biconvex set $K_{k \beta}$.

Theorem 3.1. Let $F$ be a differentiable function on the biconvex set $K_{k \beta}$ in $H$ and let the condition $M$ hold. Then the function $F$ is higher order generalized biconvex function, if and only if,

$$
F(v)-F(u) \geq F_{k \beta}^{\prime}(u, \beta(v-u))+\mu\|\beta(v-u)\|^{p}, \forall u, v \in K_{k \beta}, t \in[0,1], p>1 .
$$


Proof. Let $f$ be a higher order generalized biconvex function on biconvex set $K_{k \beta}$. Then,

$$
\begin{aligned}
& F(u+k(\lambda) \beta(v-u)) \leq(1-k(\lambda)) F(u)+\lambda F(v) \\
& -\mu\left\{k(\lambda)^{p}(1-k(\lambda))+k(\lambda)(1-k(\lambda))^{p}\right\}\|\beta(v-u)\|^{p}, \\
& \forall u, v \in K_{k \beta}, \lambda \in[0,1], p \geq 1,
\end{aligned}
$$

which can be written as:

$$
\begin{aligned}
& F(v)-F(u) \geq\left\{\frac{F(u+\lambda \beta(v-u))-F(u)}{k(\lambda)}\right\} \\
& +\mu\left\{k(\lambda)^{p}(1-k(\lambda))+k(\lambda)(1-k(\lambda))^{p}\right\}\|\beta(v-u)\|^{p} .
\end{aligned}
$$

Taking the limit in the above inequality as $\lambda \rightarrow 0$, we have

$$
F(v)-F(u) \geq F_{k \beta}^{\prime}(u, \beta(v-u))+\mu\|\beta(v-u)\|^{p},
$$

which is the required (20).

Conversely, let $F$ satisfy (20). Then, $\forall u, v \in K_{k \beta}, \lambda \in[0,1]$, $v_{\lambda}=u+k(\lambda) \beta(v-u) \in K_{k \beta}$ and using the condition $M$, we have

$$
\begin{aligned}
& F(v)-F(u+k(\lambda) \beta(v-u)) \\
\geq & \left\langle F^{\prime}(u+k(\lambda) \beta(v-u)), \eta(v, u+k(\lambda) \beta(v-u))\right\rangle \\
& +\mu\|\eta(v-u-k(\lambda) \beta(v-u))\|^{p} \\
= & (1-k(\lambda))\left\langle F_{k \beta}^{\prime}(u+k(\lambda) \beta(v-u)), \beta(v-u)\right\rangle \\
& +\mu(1-k(\lambda))^{p}\|\beta(v-u)\|^{p} .
\end{aligned}
$$

In a similar way, we have

$$
\begin{aligned}
& F(u)-F(u+k(\lambda) \beta(v-u)) \\
\geq & \left\langle F_{k \beta}^{\prime}(u+k(\lambda) \beta(v-u)), \beta(u-u-k(\lambda) \beta(v-u))\right\rangle \\
& +\mu\|\eta(u-u-k(\lambda) \beta(v-u))\|^{p} \\
= & -k(\lambda)\left\langle F_{k \beta}^{\prime}(u+k(\lambda) \beta(v-u)), \beta(v-u)\right\rangle+\mu k(\lambda)^{p}\|\beta(v-u)\|^{p} .
\end{aligned}
$$

Multiplying (3.2) by $k(\lambda)$ and (3.3) by $(1-k(\lambda))$ and adding the resultant, we have

$$
\begin{aligned}
& F(u+k(\lambda) \beta(v-u)) \leq(1-k(\lambda)) F(u)+k(\lambda) F(v) \\
& -v\left\{(k(\lambda))^{p}(1-k(\lambda))+k(\lambda)(1-k(\lambda))^{p}\right\}\|\beta(v-u)\|^{p},
\end{aligned}
$$

showing that $F$ is a higher order strongly biconvex function.

Theorem 3.2. Let $F$ be differentiable higher order generalized biconvex function on the biconvex set $K_{k \beta}$. If $F$ is a higher order strongly biconvex function, then,

$$
\begin{aligned}
& F_{k \beta}^{\prime}(u, \beta(v-u))+F_{k \beta}^{\prime}(v, \beta(u-v)) \\
& \leq-\mu\left\{\|\beta(v-u)\|^{p}+\|\beta(u-v)\|^{p}\right\}, \forall u, v \in K_{k \beta} .
\end{aligned}
$$


Proof. Let $F$ be a higher order strongly biconvex function on the biconvex set $K_{k \beta}$. Then,

$$
F(v)-F(u) \geq F_{k \beta}^{\prime}(u, \beta(v-u))+\mu\|\beta(v-u)\|^{p}, \quad \forall u, v \in K_{k \beta} .
$$

Changing the role of $u$ and $v$ in (24), we have

$$
F(u)-F(v) \geq F_{k \beta}^{\prime}(v, \beta(u-v))+\mu\|\beta(u-v)\|^{p}, \quad \forall u, v \in K_{k \beta} .
$$

Adding (24) and (25), we have

$$
\begin{aligned}
& F_{k \beta}^{\prime}(u, \beta(v-u))+F_{k \beta}^{\prime}(v, \beta(u-v)) \\
& \leq-\mu\left\{\|\beta(v-u)\|^{p}+\|\beta(u-v)\|^{p}\right\}, \forall u, v \in K_{k \beta} .
\end{aligned}
$$

which shows that $F_{k \beta}^{\prime}($.$) is a higher order strongly monotone operator.$

Theorem 3.3. If the differential $F_{k \beta}^{\prime}($.$) is a higher order strongly monotone,$ then,

$$
F(v)-F(u) \geq F_{k \beta}^{\prime}(u, \beta(v-u))+\mu\|\beta(v-u)\|^{p} \int_{0}^{1} k(\lambda)^{p-1} \mathrm{~d} \lambda .
$$

Proof. Let $F_{k \beta}^{\prime}($.$) be a higher order strongly k \beta$-monotone. From (26), we have

$$
F_{k \beta}^{\prime}(v, \beta(u-v)) \geq F_{k \beta}^{\prime}(u, \beta(v-u))-\mu\left\{\|\beta(v-u)\|^{p}+\|\beta(u-v)\|^{p}\right\} .
$$

Since $K$ is an biconvex set, $\forall u, v \in K_{k \beta}, \quad \lambda \in[0,1], \quad v_{\lambda}=u+k(\lambda) \beta(v-u) \in K_{k \beta}$.

Taking $v=v_{\lambda}$ in (27) and using Condition $M$, we have

$$
\begin{aligned}
& F_{k \beta}^{\prime}\left(v_{\lambda}, \beta(u, u+k(\lambda) \beta(v-u))\right) \\
& \leq F_{k \beta}^{\prime}(u, \beta(u+k(\lambda) \beta(v-u), u)) \\
& \quad-\mu\left\{\|\beta(u+k(\lambda) \beta(v-u), u)\|^{p}+\|\beta(u, u+k(\lambda) \beta(v-u))\|^{p}\right\} \\
& =-k(\lambda) F_{k \beta}^{\prime}(u, \beta(v-u))-2 k(\lambda)^{p} \mu\|\beta(v-u)\|^{p},
\end{aligned}
$$

which implies that

$$
F_{k \beta}^{\prime}\left(v_{\lambda}, \beta(v-u)\right) \geq F_{k \beta}^{\prime}(u, \beta(v-u))+2 \mu k(\lambda)^{p-1}\|\beta(v-u)\|^{p} .
$$

Let $\xi(\lambda)=F(u+k(\lambda) \beta(v-u)$. Then, from (28), we have

$$
\begin{aligned}
\xi^{\prime}(\lambda) & =F_{k}^{\prime}(u+k(\lambda) \beta(v-u), \beta(v-u)) \\
& \geq F_{k \beta}^{\prime}(u, \beta(v-u))+2 \mu k(\lambda)^{p-1}\|\beta(v-u)\|^{p} .
\end{aligned}
$$

Integrating (3.10) between 0 and 1, we have

$$
\xi(1)-\xi(0) \geq F_{k \beta}^{\prime}(u, \beta(v-u))+\mu\|\beta(v-u)\|^{p} \int_{0}^{1} k(\lambda)^{p-1} \mathrm{~d} \lambda .
$$

that is

$$
F(u+k(1) \beta(v-u))-F(u) \geq F_{k \beta}^{\prime}(u, \beta(v-u))+\mu\|\beta(v-u)\|^{p} \int_{0}^{1} k(\lambda)^{p-1} \mathrm{~d} \lambda .
$$


By using Condition A, we have

$$
F(v)-F(u) \geq F_{k \beta}^{\prime}(u, \beta(v-u))+\mu\|\beta(v-u)\|^{p} \int_{0}^{1} k(\lambda)^{p-1} \mathrm{~d} \lambda .
$$

the required result.

We now give a necessary condition for higher order strongly pseudo-biconvex function.

Theorem 3.4. Let $F_{k \beta}^{\prime}($.$) be a higher order strongly relaxed k$-pseudomonotone operator and Conditions $A$ and $M$ hold. Then

$$
F(v)-F(u) \geq \alpha\|\eta(v, u)\|^{p} \int_{0}^{1} k(\lambda) \mathrm{d} \lambda,
$$

Proof. Let $F^{\prime}$ be higher order relaxed $k$-pseudomonotone. Then, $\forall u, v \in K_{k \eta}$,

$$
F_{k \beta}^{\prime}(u, \beta(v-u)) \geq 0
$$

implies that

$$
-F_{k \beta}^{\prime}(v, \beta(u-v)) \geq \alpha\|\beta(u-v)\|^{p} .
$$

Since $K_{k \beta}$ is an biconvex set, $\forall u, v \in K_{k \beta}, \lambda \in[0,1]$, $v_{\lambda}=u+k(\lambda) \beta(v-u) \in K_{k \beta}$.

Taking $v=v_{\lambda}$ in (3.12) and using condition Condition $M$, we have

$$
-F_{k \beta}^{\prime}(u+\lambda \beta(v-u), \beta(u-v)) \geq k(\lambda) \alpha\|\beta(v-u)\|^{p} .
$$

Let

$$
\xi(\lambda)=F(u+k(\lambda) \beta(v-u)), \quad \forall u, v \in K_{k \beta}, \lambda \in[0,1]
$$

Then, using (3.13), we have

$$
\xi^{\prime}(\lambda)=F_{k \beta}^{\prime}(u+k(\lambda) \beta(v-u), \beta(u-v)) \geq k(\lambda) \alpha\|\beta(v-u)\|^{p} .
$$

Integrating the above relation between 0 to 1 , we have

$$
\xi(1)-\xi(0) \geq \alpha\|\beta(v-u)\|^{p} \int_{0}^{1} k(\lambda) \mathrm{d} \lambda,
$$

that is,

$$
F(u+k(1) \beta(v-u))-F(u) \geq \alpha\|\beta(v-u)\|^{p} \int_{0}^{1} k(\lambda) \mathrm{d} \lambda,
$$

which implies, using Condition A,

$$
F(v)-F(u) \geq \alpha\|\beta(v-u)\|^{p} \int_{0}^{1} k(\lambda) \mathrm{d} \lambda,
$$

which is the required (3.11).

Theorem 3.5. Let the differential $\mathrm{F}^{\prime}(u)$ of a differentiable biconvex function $F(u)$ be Lipschitz continuous on the biconvex set $K_{k \beta}$ with a constant $\beta>0$. Then, 


$$
F(u+\beta(v-u))-F(u) \leq F_{k \beta}^{\prime}(u, \eta(v, u))+\beta\|\beta(v-u)\|^{p} \int_{0}^{1} k(\lambda) \mathrm{d} \lambda, \quad u, v \in K_{k \beta}
$$

Proof. Its proof follow from Noor and Noor [22].

Definition 3.1. The function $F$ is said to be sharply higher order strongly pseudo biconvex, if there exists a constant $\mu>0$ such that

$$
\begin{gathered}
F_{k \beta}^{\prime}(u, \beta(v-u)) \geq 0 \\
\Rightarrow \\
F(v) \geq F(v+k(\lambda) \beta(v-u))+\mu\left\{k(\lambda)^{p}(1-k(\lambda))+\lambda(1-k(\lambda))^{p}\right\}\|\beta(v-u)\|^{p}, \\
\forall u, v \in K_{k \beta}, \lambda \in[0,1] .
\end{gathered}
$$

Theorem 3.6. Let $F$ be a sharply higher order strongly pseudo biconvex function on $K_{k \beta}$ with a constant $\mu>0$. Then

$$
-F_{k \beta}^{\prime}(v, \beta(v-u)) \geq \mu\|\beta(v-u)\|^{p}, \quad \forall u, v \in K_{k \beta} .
$$

Proof. Let $F$ be a sharply higher strongly pseudo generalized biconvex function on $K_{\eta}$. Then

$$
\begin{aligned}
& F(v) \geq F(v+k(\lambda) \beta(v-u)) \\
& +\mu\left\{k(\lambda)^{p}(1-k(\lambda))+k(\lambda)(1-k(\lambda))^{p}\right\}\|\beta(v-u)\|^{p}, \\
& \forall u, v \in K_{k \beta}, \lambda \in[0,1],
\end{aligned}
$$

from which we have

$$
\begin{aligned}
& \frac{F(v+k(\lambda) \beta(v-u))-F(v)}{k(\lambda)} \\
& +\mu\left\{k(\lambda)^{p-1}(1-k(\lambda))+(1-k(\lambda))^{p}\right\}\|\beta(v-u)\|^{p} \leq 0 .
\end{aligned}
$$

Taking limit in the above-mentioned inequality, as $\lambda \rightarrow 0$, we have

$$
-F_{k \beta}^{\prime}(v, \beta(v-u)) \geq \mu\|\eta(v, u)\|^{p}, \quad \forall u, v \in K_{k \beta},
$$

the required result.

Definition 3.2. A function $F$ is said to be a higher order strongly pseudo biconvex function with respect to strictly positive bifunction $W(.,$.$) , if$

$$
\begin{gathered}
F(v)<F(u) \\
\Rightarrow \\
F(u+k(\lambda) \beta(v-u))<F(u)+k(\lambda)(k(\lambda)-1) W(v, u), \forall u, v \in K_{k \beta}, \lambda \in[0,1] .
\end{gathered}
$$

Theorem 3.7. If the function $F$ is higher order strongly biconvex function such that $F(v)<F(u)$, then the function $F$ is higher order strongly pseudo biconvex.

Proof. Since $F(v)<F(u)$ and $F$ is higher order strongly biconvex function, then $\forall u, v \in K_{k \eta}, \lambda \in[0,1]$, we have 


$$
\begin{aligned}
& F(u+k(\lambda) \beta(v-u)) \\
\leq & F(u)+k(\lambda)(F(v)-F(u)) \\
& -\mu\left\{k(\lambda)^{p}(1-k(\lambda))+k(\lambda)(1-k(\lambda))^{p}\right\}\|\beta(v-u)\|^{p} \\
< & F(u)+k(\lambda)(1-k(\lambda))(F(v)-F(u)) \\
& -\mu\left\{k(\lambda)^{p}(1-k(\lambda))+k(\lambda)(1-k(\lambda))^{p}\right\}\|\beta(v-u)\|^{p} \\
= & F(u)+k(\lambda)(k(\lambda)-1)(F(u)-F(v)) \\
& -\mu\left\{k(\lambda)^{p}(1-k(\lambda))+k(\lambda)(1-k(\lambda))^{p}\right\}\|\beta(v-u)\|^{p} \\
< & F(u)+k(\lambda)(k(\lambda)-1) W(u, v) \\
& -\mu\left\{k(\lambda)^{p}(1-k(\lambda))+k(\lambda)(1-k(\lambda))^{p}\right\}\|\beta(v-u)\|^{p}, \forall u, v \in K_{k \beta} .
\end{aligned}
$$

where $W(u, v)=F(u)-F(v)>0$. This shows that the function $F$ is higher order strongly pseudo biconvex.

\section{Applications}

In this section, we derive new parallelogram laws for uniformly Banach spaces as a novel application of higher order strongly biconvex functions.

From definition 2.13, we have

$$
\begin{aligned}
& F(u+k(\lambda) \beta(v-u))=(1-k(\lambda)) F(u)+k(\lambda) F(v) \\
& -\mu\left\{k(\lambda)^{p}(1-k(\lambda))+k(\lambda)(1-k(\lambda))^{p}\right\}\|\beta(v-u)\|^{p}, \\
& \forall u, v \in K_{k \beta}, \lambda \in[0,1], p \geq 1 .
\end{aligned}
$$

Taking $\lambda=\frac{1}{2}$ in (4.1), we have

$$
\begin{aligned}
& F\left(u+k\left(\frac{1}{2}\right) \beta(v-u)\right) \\
& +\mu\left\{\left(k\left(\frac{1}{2}\right)\right)^{p}\left(1-k\left(\frac{1}{2}\right)\right)+k\left(\frac{1}{2}\right)\left(1-k\left(\frac{1}{2}\right)\right)^{p}\right\}\|\beta(v-u)\|^{p} \\
& =k\left(\frac{1}{2}\right) F(u)+k\left(\frac{1}{2}\right) F(v), \forall u, v \in K_{\eta} .
\end{aligned}
$$

which is known as the generalized parallelogram-like laws for the Banach spaces involving higher order generalized biconvex functions.

We now discuss some special cases of the generalized parallelogram-like laws.

1) If $k\left(\frac{1}{2}\right)=\frac{1}{2}, F(u)=\|u\|^{p}, p>1$, then, Equation (4.2) becomes

$$
\left\|u+\frac{1}{2} \beta(v-u)\right\|^{p}+\mu \frac{1}{2^{p}}\|\beta(v-u)\|^{p}=\frac{1}{2}\|u\|^{p}+\frac{1}{2}\|v\|^{p}, \forall u, v \in K_{k \beta} .
$$

2) If $\beta(v, u)=v-u, k\left(\frac{1}{2}\right)=\frac{1}{2}$, then (4.3) reduces to the parallelogram-like law as: 


$$
\|v+u\|^{p}+\mu\|v-u\|^{p}=2^{p-1}\left\{\|u\|^{p}+\|v\|^{p}\right\},
$$

which is known as the parallelogram-like law for the uniform Banach spaces involving the biconvex functions. $\mathrm{Xu}$ [36] obtained these characterizations of $p$ uniform convexity and $q$-uniform smoothness of a Banach space via the functionals $\|\cdot\|^{p}$ and $\|\cdot\|^{q}$, respectively. Bynum [37] and Chen et al. [38] [39] have studied the properties and applications of the parallelogram laws for the Banach spaces. For the applications of the parallelogram laws in Banach spaces in prediction theory and applied sciences, see [36] [37] [38] [39] and the references therein.

3) If $p=2, K\left(\frac{1}{2}\right)=\frac{1}{2}$, then parallelogram law (4.2) reduces to:

$$
\|2 u+\beta(v-u)\|^{2}+\mu\|\beta(v-u)\|^{2}=2\left\{\|u\|^{2}+\|v\|^{2}\right\} .
$$

This is a new parallelogram law, which characterizes the inner product spaces involving bifunction $\beta(.-$.$) .$

4) If $\beta(v, u)=v-u, \mu=1$, then parallelogram law (37) becomes

$$
\|v+u\|^{2}+\|v-u\|^{2}=2\left\{\|u\|^{2}+\|v\|^{2}\right\}
$$

which is a well known parallelogram and characterizes the inner product spaces.

Remark 4.1. In this section, we have derived some new parallelogram laws as important applications of the higher order strongly biconvex functions. For suitable and appropriate choice of the function $k$, bifunction $\beta(.-$.$) and pa-$ rameter $p$, one can obtain a wide class of parallelogram laws, which can be used to characterize various classes of inner products. Applications of the new parallelogram laws in different areas are an open problem for future research.

\section{Biequilibrium Problems}

In this section, we introduce the biequilibrium problems. We also use the auxiliary principle techniques coupled with Bregman functions to consider the convergence criteria of the proposed iterative methods. This is the main motivation of this section.

For the readers convenience, we recall some basic properties of the Bregman [27] distance functions on the convex set $K$. For strongly convex function $F$, we define the Bregman distance function as:

$$
B(v, u)=F(v)-F(u)-\left\langle F^{\prime}(u), v-u\right\rangle \geq \alpha\|v-u\|^{2}, \forall u, v \in K .
$$

It is important to emphasize that various types of function $F$ gives different Bregman distance. We give the following important examples of some practical important types of function $F$ and their corresponding Bregman distance, see [2] [3].

\section{Examples}

1) If $f(v)=\|v\|^{2}$, then $B(v, u)=\|v-u\|$, which is the squared Euclidean distance $(S E)$. 
2) If $f(v)=\sum_{i=1}^{n} a_{i} \log \left(v_{i}\right)$, which is known as Shannon entropy, then its corresponding Bregman distance is given as:

$$
B(v, u)=\sum_{i=1}^{n}\left(v_{i} \log \left(\frac{v_{i}}{u_{i}}\right)+u_{i}-v_{i}\right) .
$$

This distance is called KullbackLeibler distance $(K L)$ and as become a very important tool in several areas of applied mathematics such as machine learning.

3) If $f(v)=-\sum_{i=1}^{n} \log \left(v_{i}\right)$, which is called Burg entropy, then its corresponding Bregman distance is given as:

$$
B(v, u)=\sum_{i=1}^{n}\left(\log \left(\frac{v_{i}}{u_{i}}\right)+\frac{v_{i}}{u_{i}}-1\right) .
$$

This is called ItakuraSaito distance (IS), which is very important in information theory.

It is a challenging problem to explore the applications of Bregman distance for other types of nonconvex functions as biconvex, $k$-convex functions and harmonic functions.

We now discuss the optimality for the differentiable higher order generalized biconvex functions.

Theorem 5.1. Let $F$ be a differentiable higher order strongly biconvex function with modulus $\mu>0$. If $u \in K_{k \beta}$ is the minimum of the function $F$, then

$$
F(v)-F(u) \geq \mu\|\beta(v-u)\|^{p}, \quad \forall u, v \in K_{k \beta} .
$$

Proof. Let $u \in K_{k \beta}$ be a minimum of the function $F$. Then,

$$
F(u) \leq F(v), \forall v \in K_{k \beta} .
$$

Since $K_{k \beta}$ is an biconvex set, so, $\forall u, v \in K_{k \beta}, \lambda \in[0,1]$, $v_{\lambda}=u+k(\lambda) \beta(v-u) \in K_{k \beta}$.

Taking $v=v_{\lambda}$ in (5.3), we have

$$
0 \leq \lim _{\lambda \rightarrow 0}\left\{\frac{F(u+k(\lambda) \beta(v-u))-F(u)}{k(\lambda)}\right\}=F_{k \beta}^{\prime}(u, \beta(v-u)) .
$$

Since $F$ is differentiable higher order strongly biconvex function, so

$$
\begin{aligned}
& F(u+k(\lambda) \beta(v-u)) \leq F(u)+k(\lambda)(F(v)-F(u)) \\
& -\mu\left\{k(\lambda)^{p}(1-k(\lambda))+k(\lambda)(1-k(\lambda))^{p}\right\}\|\beta(v-u)\|^{p}, \forall u, v \in K_{k \beta} .
\end{aligned}
$$

from which, using (5.4), we have

$$
\begin{aligned}
F(v)-F(u) \geq & \lim _{\lambda \rightarrow 0}\left\{\frac{F(u+k(\lambda) \beta(v-u))-F(u)}{k(\lambda)}\right\} \\
& +\mu\left\{k(\lambda)^{p-1}(1-k(\lambda))+(1-k(\lambda))^{p}\right\}\|\beta(v-u)\|^{p} \\
= & F_{k \beta}^{\prime}(u, \beta(v-u))+\mu\|\beta(v-u)\|^{p},
\end{aligned}
$$

the required result (5.2). 
We would like to mention that, if $u \in K_{k \beta}$ satisfies the inequality,

$$
F_{k \beta}^{\prime}(u, \beta(v-u))+\mu\|\beta(v-u)\|^{p} \geq 0, \quad \forall u, v \in K_{k \beta},
$$

then $u \in K_{k \beta}$ is the minimum of the function $F$. The inequality of the type (5.5) is called the higher order strongly biequilibrium problem and appears to new one.

It is worth mentioning that inequalities of the type (5.5) may not arise as the minimization of the higher order strongly biconvex functions. This motivated us to consider a more general higher order strongly biequilibrium problem of which (5.5) is a special case.

To be more precise, for given operator $D$, bifunction $\beta(.-$.$) and a constant$ $\mu>0$, consider the problem of finding $u \in K_{k \beta}$, such that

$$
D(u, \beta(v-u))+\mu\|\beta(v-u)\|^{p} \geq 0, \forall v \in K_{k \beta}, p \geq 1,
$$

which is called the higher order strongly biequilibrium problem.

1) If $\mu=0$, then the higher order strongly biequilibrium problem (2.1) reduces to finding $u \in K_{k \beta}$ such that

$$
D(u, \beta(v-u)) \geq 0, \quad \forall v \in K_{k \beta},
$$

which is called the biequilibrium problem. For the formulation, numerical methods and other aspects of the bivariational inequalities and biequilibrium problem, see [28] [30].

2) If $\mathrm{f} D(u, \beta(v-u))=\langle A u, \beta(v-u)\rangle$, where $A$ is a nonlinear operator, then the higher order strongly biequilibrium problem (2.1) is equivalent to finding $u \in K_{k \beta}$ such that

$$
\langle A u, \beta(v-u)\rangle+\mu\|\beta(v-u)\|^{p} \geq 0, \quad \forall v \in K_{k \eta},
$$

which is the higher order strongly bivariational inequality and appears to be a new one.

It is worth mentioning that for suitable and appropriate choice of the operators, $k \beta$-biconvex sets and spaces, one can obtain a wide class of equilibrium problem, variational-like inequalities and optimization programming. This shows that the higher order strongly biequilibrium problems are quite flexible and unified ones.

Due to the inherent nonlinearity, the projection method and its variant form can not be used to suggest the iterative methods for solving these higher order strongly biequilibrium problems. To overcome these drawback, one may use the auxiliary principle technique of Glowinski et al. [11] as developed by Noor [26] [28] [30] Noor et al. [14] [15] [16] to suggest and analyze some iterative methods for solving the higher order strongly biequilibrium problems (5.6). This technique does not involve the concept of the projection, which is the main advantage of this technique.

We again use the auxiliary principle technique coupled with Bergman distance functions. These applications are based on the type of convex functions asso- 
ciated with the Bregman distance function. We now suggest and analyze some iterative methods for higher order strongly biequilibrium problems (5.6) using the auxiliary principle technique coupled with Bregman functions as developed by Noor [28] [30].

For a given $u \in K_{k \beta}$ satisfying the biequilibrium problem (5.6), we consider the auxiliary problem of finding a $w \in K_{k \beta}$ such that

$$
\begin{aligned}
& \rho D(w, \beta(v-w))+\left\langle E_{k \beta}^{\prime}(w)-E_{k \beta}^{\prime}(u), \beta(v-w)\right\rangle \\
& +\rho\|\beta(v-w)\|^{p} \geq 0, \forall v \in K_{k \beta},
\end{aligned}
$$

where $\rho>0$ is a constant and $E_{k \beta}^{\prime}(u)$ is the differential of a strongly biconvex function $E(u)$ at $u \in K$.

Remark 3.1: The function $B(w, u)=E(w)-E(u)-E_{k \beta}^{\prime}(u, \beta(w-u))$ associated with the biconvex function $E(u)$ is called the generalized Bregman function. By the strongly biconvexity of the function $E(u)$, the Bregman function $B(.,$.$) is nonnegative and B(w, u)=0$, if and only if $u=w, \forall u, w \in K_{k \beta}$. For the applications of the Bregman function in solving variational inequalities and complementarity problems, see [28] [30].

We note that, if $w=u$, then clearly $w$ is solution of the higher order strongly biequilibrium problem (5.6). This observation enables us to suggest and analyze the following iterative method for solving (2.1).

Algorithm 3.1. For a given $u_{0} \in H$, compute the approximate solution $u_{n+1}$ by the iterative scheme:

$$
\begin{aligned}
& \rho D\left(u_{n+1}, \beta\left(v-u_{n+1}\right)\right)+\left\langle E_{k \beta}^{\prime}\left(u_{n+1}\right)-E_{k \beta}^{\prime}\left(u_{n}, \beta\left(v-u_{n+1}\right)\right)\right\rangle \\
& +\rho\|\beta(v-u)\|^{p} \geq 0, \quad \forall v \in K_{k \beta},
\end{aligned}
$$

where $\rho>0$ is a constant. Algorithm 3.1 is called the proximal method for solving higher order strongly biequilibrium-like problem (5.6). In passing we remark that the proximal point method was suggested in the context of convex programming problems as a regularization technique.

If $\mu=0$, then Algorithm 3.1 collapses to:

Algorithm 3.2. For a given $u_{0} \in H$, compute the approximate solution $u_{n+1}$ by the iterative scheme:

$$
\rho D\left(u_{n+1}, \beta\left(v-u_{n+1}\right)\right)+\left\langle E_{k \beta}^{\prime}\left(u_{n+1}\right)-E_{k \beta}^{\prime}\left(u_{n}, \beta\left(v-u_{n+1}\right)\right)\right\rangle \geq 0, \quad \forall v \in K_{k \beta},
$$

for solving the bivariational inequality.

For suitable and appropriate choice of the operators and the spaces, one can obtain a number of known and new algorithms for solving variational inequalities and related problems.

Definition 2.2. The bifunction $D(.,$.$) is said to be:$

1) monotone, iff,

$$
D(u, \beta(v-u))+D(v, \beta(u-v)) \leq 0, \quad \forall u, v \in K_{k \beta} .
$$

2) pseudomonotone with respect to $\mu\|\beta(v-u)\|^{p}$, iff, 


$$
\begin{gathered}
D(u, \beta(v-u))+\mu\|\beta(v-u)\|^{p} \geq 0 \\
\Rightarrow \\
-D(v, \beta(u-v))-\mu\|\beta(v-u)\|^{p} \geq 0, \quad \forall u, v \in K_{k \beta} .
\end{gathered}
$$

3) higher order strongly partially relaxed monotone, if there exists a constant $\gamma>0$ such that

$$
D(u, \beta(u-v))+D(v, \beta(z-v)) \leq \gamma\|\beta(z-u)\|^{p}, \quad \forall u, v, z \in K_{k \beta} .
$$

Note that for $z=u$ higher order strongly partially relaxed monotonicity reduces to monotonicity. This shows that higher order strongly partially relaxed monotonicity implies monotonicity, but the converse is not true.

Theorem 5.2. Let the bifunction $D(.,$.$) be pseudomonotone with respect to$ $\mu\|\beta(v-u)\|^{p}$. If $E$ be differentiable higher order strongly biconvex function with module $\beta>0$, then the approximate solution $u_{n+1}$ obtained from Algorithm 3.1 converges to a solution $u \in K$ satisfying the biequilibrium problem (5.6).

Proof. Let $u \in K_{k \beta}$ be a solution of (5.6). Then,

$$
D(u, \beta(v-u))+\mu\|\beta(v-u)\|^{p} \geq 0, \quad \forall v \in K_{k \beta},
$$

implies that

$$
-D(v, \beta(u-v))-\mu\|\beta(v-u)\|^{p} \geq 0, \quad \forall v \in K_{k \beta},
$$

since $D(.,$.$) is pseudomonotone with respect to \mu\|\beta(v-u)\|^{p}$.

Taking $v=u$ in (5.8) and $v=u_{n+1}$ in (5.9), we have

$$
\begin{aligned}
& \rho D\left(u_{n+1}, \beta\left(u-u_{n+1}\right)\right)+\left\langle E_{k \beta}^{\prime}\left(u_{n+1}\right)-E_{k \beta}^{\prime}\left(u_{n}, \beta\left(u-u_{n+1}\right)\right)\right\rangle \\
& \geq-\rho \mu\left\|\beta\left(u-u_{n+1}\right)\right\|^{p} .
\end{aligned}
$$

And,

$$
-D\left(u_{n+1}, \beta\left(u-u_{n+1}\right)\right)-\mu\left\|\beta\left(u-u_{n+1}\right)\right\|^{p} \geq 0 .
$$

We now consider the Bregman function

$$
B(u, w)=E(u)-E(w)-\left\langle E_{k \beta}^{\prime}(w, \beta(u-w))\right\rangle \geq \mu\|\beta(u-w)\|^{p},
$$

using higher order strongly biconvexity of $E$.

Now combining (5.10), (5.11) and (5.12), we have

$$
\begin{aligned}
& B\left(u, u_{n}\right)-B\left(u, u_{n+1}\right) \\
& =E\left(u_{n+1}\right)-E\left(u_{n}\right)-\left\langle E_{k \beta}^{\prime}\left(u_{n}, \beta\left(u-u_{n}\right)\right)\right\rangle+\left\langle E_{k \beta}^{\prime}\left(u_{n+1}, \beta\left(u-u_{n+1}\right)\right)\right\rangle \\
& =E\left(u_{n+1}\right)-E\left(u_{n}\right)-\left\langle E_{k \beta}^{\prime}\left(u_{n}\right)-E_{k \beta}^{\prime}\left(u_{n+1}, \beta\left(u-u_{n+1}\right)\right)\right\rangle-E_{k \beta}^{\prime}\left(u_{n}, \beta\left(u_{n+1}-u_{n}\right)\right) \\
& \geq \mu\left\|\beta\left(u_{n+1}-u_{n}\right)\right\|^{p}+\left\langle E_{k \beta}^{\prime}\left(u_{n+1}\right)-E_{k \beta}^{\prime}\left(u_{n}, \beta\left(u-u_{n+1}\right)\right)\right\rangle \\
& \geq \mu\left\|\beta\left(u_{n+1}-u_{n}\right)\right\|^{p}-\rho D\left(u_{n+1}, \beta\left(u-u_{n+1}\right)\right)-\rho \mu\left\|\beta\left(u-u_{n+1}\right)\right\|^{p} \\
& \geq \mu\left\|\beta\left(u_{n+1}-u_{n}\right)\right\|^{p} .
\end{aligned}
$$


If $u_{n+1}=u_{n}$, then clearly $u_{n}$ is a solution of the problem (5.6). Otherwise, it follows that $B\left(u, u_{n}\right)-B\left(u, u_{n+1}\right)$ is nonnegative and we must have

$$
\lim _{n \rightarrow \infty}\left(\beta\left(u_{n+1}-u_{n}\right)\right)=0 .
$$

from which, we have

$$
\lim _{n \rightarrow \infty}\left\|u_{n+1}-u_{n}\right\|=0 .
$$

It follows that the sequence $\left\{u_{n}\right\}$ is bounded. Let $\bar{u}$ be a cluster point of the subsequence $\left\{u_{n_{i}}\right\}$, and let $\left\{u_{n_{i}}\right\}$ be a subsequence converging toward $\bar{u}$. Now using the technique of Zhu and Marcotte [17], it can be shown that the entire sequence $\left\{u_{n}\right\}$ converges to the cluster point $\bar{u}$ satisfying the biequilibrium problem (5.6).

It is well-known that to implement the proximal point methods, one has to find the approximate solution implicitly, which is itself a difficult problem. To overcome this drawback, we now consider another method for solving the higher order strongly biequilibrium problem (5.6) using the auxiliary principle technique.

For a given $u \in K_{k \beta}$, find $w \in K_{k \beta}$, such that

$$
\begin{aligned}
& \rho D(u, \beta(v-w))+\left\langle E_{k \beta}^{\prime}(w)-E_{k \beta}^{\prime}(u), \beta(v-w)\right\rangle \\
& +\rho \mu\|\beta(v-w)\|^{p} \geq 0, \quad \forall v \in K_{k \beta},
\end{aligned}
$$

where $E_{k}^{\prime}(u)$ is the differential of a strongly $k$-biconvex function $E(u)$ at $u \in K_{k \beta}$. Note that problems (5.13) and (5.8) are quite different problems. It is clear that for $w=u, w$ is a solution of (5.6). This fact allows us to suggest and analyze another iterative method for solving the higher order strongly biequilibrium problem (5.6).

Algorithm 3.3. For a given $u_{0} \in H$, compute the approximate solution $u_{n+1}$ by the iterative scheme:

$$
\begin{aligned}
& \rho D\left(u_{n}, \beta\left(v-u_{n+1}\right)\right)+\left\langle E_{k \beta}^{\prime}\left(u_{n+1}\right)-E_{k \beta}^{\prime}\left(u_{n}\right), \beta\left(v-u_{n+1}\right)\right\rangle \\
& \geq-\rho \mu\left\|\beta\left(v-u_{n+1}\right)\right\|^{p}, \quad \forall v \in K_{k \beta},
\end{aligned}
$$

for solving the higher order strongly biequilibrium problem (5.6).

If $D(u, \beta(v, u))=\langle A u, \beta(v-u)\rangle$, Algorithm 3.3 collapses to:

Algorithm 3.4. For a given $u_{0} \in H$, compute the approximate solution $u_{n+1}$ by the iterative schemes:

$$
\begin{aligned}
& \rho\left\langle A u_{n}, \beta\left(v-u_{n+1}\right)\right\rangle+\left\langle E_{k \beta}^{\prime}\left(u_{n+1}\right)-E_{k \beta}^{\prime}\left(u_{n}\right), \beta\left(v-u_{n+1}\right)\right\rangle \\
& \geq-\rho \mu \|\left.\beta\left(v-u_{n+1}\right)\right|^{p}, \quad \forall v \in K_{k \beta},
\end{aligned}
$$

for solving the higher order strongly bivariational inequalities and appears to be a new one.

Discussion of Results: We have introduced and investigated higher order strongly biequilibrium problems. The auxiliary principle technique coupled with Bregman distance functions is used to suggest some iterative methods for solv- 
ing biequilibrium problems, which is the main contributions of this section. We also mention that for suitable and appropriate choice of the operators and the spaces, one can obtain various known and new algorithms for solving higher order strongly equilibrium problem (5.6) and related optimization problems. It is an interesting problem from both analytically and numerically point of view.

\section{Conclusion}

In this paper, we have introduced and studied some new classes of biconvex functions, which are called higher order strongly biconvex functions. These concepts are more general and unifying than the previous ones. Several new properties of these higher order strongly biconvex functions are discussed and their relations with previously known results are highlighted. Several parallelogram laws for inner product spaces and Banach spaces are obtained as applications of the higher order strongly $k$-convex functions, which is its can viewed as novel applications of higher order strongly biconvex functions. It is shown that the optimality conditions of the differentiable $k$-biconvex functions can be characterized by a class of higher order strongly biequilibrium problems. This result is used to introduce some new classes of higher order strongly biequlibrium problems. The auxiliary principle technique involving the Bregman functions are used to suggest and investigate some iterative methods for solving higher order strongly biequilibrium problem. These techniques include the entropy method and its variant forms. Consequently, we can conclude that the entropy methods have played an important in the analysis of the convergence criteria of the proposed methods implicitly. It is itself an interesting problem to develop some efficient numerical methods for solving higher order strongly biequilibrium problems along with their applications in pure and applied sciences. Despite the current activity, much clearly remains to be done in these fields. It is expected that the ideas and techniques of this paper may be the starting point for future research activities.

\section{Acknowledgements}

We wish to express our deepest gratitude to our colleagues, collaborators, and friends, who have direct or indirect contributions in the process of this paper. We are also like to thank the Rector, COMSATS University Islamabad, Pakistan for the research facilities and support in our research endeavors.

\section{Conflicts of Interest}

The authors declare no conflicts of interest regarding the publication of this paper.

\section{References}

[1] Stampacchia, G. (1964) Formes bilineaires coercivites sur les ensembles convexes. Comptes rendus de 1 Académie des Sciences Paris, 258, 4413-4416. 
[2] Jolaoso, L.O., Aphane, M. and Khan, S.H. (2020) Two Bregman Projection Methods for Solving Variational Inequality Problems in Hilbert Spaces with Applications to Signal Processing. Symmetry, 12, 2007. https://doi.org/10.3390/sym12122007

[3] Sunthrayuth, P. and Cholamjiak, P. (2020) Modified Extragradient Method with Bregman Distance for Variational Inequalities. Applicable Analysis.

[4] Glowinski, R., Lions, J.L. and Tremolieres, R. (1981) Numerical Analysis of Variational Inequalities. North-Holland, Amsterdam.

[5] Kinderlehrer, D. and Stampaccia, G. (1980) Introduction to Variational Inequalties and Applications. Academic Press, New York.

[6] Lin, G.H. and Fukushima, M. (2003) Some Exact Penalty Results for Nonlinear Programs and Mathematical Programs with Equilibrium Constraints. Journal of Optimization Theory and Applications, 118, 67-80. https://doi.org/10.1023/A:1024787424532

[7] Lions, J.L. and Stampacchia, G. (1967) Variational Inequalities. Communications on Pure and Applied Mathematics, 20, 493-519. https://doi.org/10.1002/cpa.3160200302

[8] Noor, M.A. (1988) General Variational Inequalities. Applied Mathematics Letters, 1, 119-121. https://doi.org/10.1016/0893-9659(88)90054-7

[9] Noor, M.A. (2000) New Approximation Schemes for General Variational Inequalities. Journal of Mathematical Analysis and Applications, 251, 217-229. https://doi.org/10.1006/jmaa.2000.7042

[10] Noor, M.A. (2006) Fundamental of Equilibrium Problems. Mathematical Inequalities \& Applications, 9, 520-566. https://doi.org/10.7153/mia-09-51

[11] Noor, M.A. (2004) Some Developments in General Variational Inequalities. Applied Mathematics and Computation, 152, 199-277.

https://doi.org/10.1016/S0096-3003(03)00558-7

[12] Noor, M.A. (1994) Variational-Like Inequalities. Optimization, 30, 323-330. https://doi.org/10.1080/02331939408843995

[13] Noor, M.A. (2005) Invex Equilibrium Problems. Journal of Mathematical Analysis and Applications, 302, 463-475. https://doi.org/10.1016/j.jmaa.2004.08.014

[14] Noor, M.A., Noor, K.I. and Al-Said, E. (2011) Auxiliary Principle Technique for Solving Bifunction Variational Inequalities. Journal of Optimization Theory and Applications, 149, 441-445. https://doi.org/10.1007/s10957-010-9785-z

[15] Noor, M.A., Noor, K.I. and Rassias, M.Th. (2020) New Trends in General Variational Inequalities. Acta Applicandae Mathematicae, 170, 981-1064.

https://doi.org/10.1007/s10440-020-00366-2

[16] Noor, M.A., Noor, K.I. and Rassias, Th.M. (1993) Some Aspects of Variational Inequalities. Journal of Applied Mathematics and Computing, 47, 485-512. https://doi.org/10.1016/0377-0427(93)90058-J

[17] Zhu, D.L. and Marcotte, P. (1966) Co-Coercvity and Its Role in the Convergence of Iterative Schemes for Solving Variational Inequalities. SIAM Journal on Optimization, 6, 714-726. https://doi.org/10.1137/S1052623494250415

[18] Blum, E. and Oettli, W. (1994) From Optimization and Variational Inequalities to Equilibrium Problems. Mathematics Student, 63, 123-145.

[19] Noor, M.A. and Oettli, W. (1994) On General Nonlinear Complementarity Problems and Quasi-Equilibria. Le Matematiche (Catania), 49, 313-331.

[20] Noor, M.A. and Noor, K.I. (2021) Exponentially Biconvex Functions and Bivaria- 
tional Inequalties. In: Hazarika, B., Acharjee, S. and Srivastava, H.M., Eds., Advances in Mathematical Analysis and Multidisciplinary Applications, CRC Press, Boca Raton, 23 p.

[21] Noor, M.A., Noor, K.I. and Rassias, M.T. (2021) Strongly Biconvex Functions and Bi-Variational Inequalities. In: Pardalos, P.M. and Rassias, Th.M., Eds., Mathematical Analysis, Optimization, Approximation and Applications, World Scientific Publishing Company, Singapore, $21 \mathrm{p}$.

[22] Noor, M.A. and Noor, K.I. (2021) Properties of Higher Order Preinvex Functions. Numerical Algebra, Control and Optimization, 11, 431-441. https://doi.org/10.3934/naco.2020035

[23] Micherda, B. and Rajba, T. (2012) On Some Hermite-Hadamard-Fejer Inequalities for (k,h)-Convex Functions. Mathematical Inequalities \& Applications, 12, 931-940. https://doi.org/10.7153/mia-15-79

[24] Hazy, A. (2012) Bernstein-Doetsch Type Results for (k,h)-Convex Functions. Miskolc Mathematical Notes, 13, 325-336. https://doi.org/10.18514/MMN.2012.538

[25] Crestescu, G., Gaianu, M. and Awan, M.U. (2015) Regularity Properties and Integral Inequalities Related to $\left(\mathrm{k}, \mathrm{h}_{1}, \mathrm{~h}_{2}\right)$-Convexity of Functions. Annals of West University of Timisoara-Mathematics and Computer Science, 53, 19-35. https://doi.org/10.1515/awutm-2015-0002

[26] Noor, M.A. (2006) Some New Classes of Nonconvex Functions. Nonlinear Functional Analysis and Applications, 11, 165-171.

[27] Bregman, L.M. (1967) The Relaxation Method for Finding Common Points of Convex Sets and Its Application to the Solution of Problems in Convex Programming. USSR Computational Mathematics and Mathematical Physics, 7, 200-217. https://doi.org/10.1016/0041-5553(67)90040-7

[28] Mohsen, B.B., Noor, M.A., Noor, K.I. and Postolache, M. (2019) Strongly Convex Functions of Higher Order Involving Bifunction. Mathematics, 7, 1028. https://doi.org/10.3390/math7111028

[29] Alabdali, O., Guessab, A. and Schmeisser, G. (2019) Characterizations of Uniform Convexity for Differentiable Functions. Applicable Analysis and Discrete Mathematics, 13, 721-732. https://doi.org/10.2298/AADM190322029A

[30] Olbrys, A. (2018) A Support Theorem for Generalized Convexity and Its Applications. Journal of Mathematical Analysis and Applications, 458, 1044-1058. https://doi.org/10.1016/j.jmaa.2017.09.038

[31] Mako, J. and Pales, Z. (2012) On $\phi$-Convexity. Publicationes Mathematicae Debrecen, 80, 107-126.

[32] Cristescu, G. and Lupsa, L. (2002) Non-Connected Convexities and Applications. Kluwer Academic Publisher, Dordrecht.

[33] Niculescu, C.P. and Persson, L.E. (2018) Convex Functions and Their Applications. Springer-Verlag, New York.

[34] Pecaric, J.E., Proschan, F. and Tong, Y.L. (1992) Convex Functions and Statistical Applications. Academic Press, New York.

[35] Tiel, J.V. (1984) Convex Analysis. John Wiley and Sons, New York.

[36] Xu, H.K. (1991) Inequalities in Banach Spaces with Applications. Nonlinear Analysis, Theory, Methods and Applications, 16, 1127-1138. https://doi.org/10.1016/0362-546X(91)90200-K

[37] Bynum, W.L. (1976) Weak Parallelogram laws for Banach Spaces. Canadian Mathematical Bulletin, 19, 269-275. https://doi.org/10.4153/CMB-1976-042-4 
[38] Cheng, R. and Harris, C.B. (2013) Duality of the Weak Parallelogram Laws on Banach Spaces. Journal of Mathematical Analysis and Applications, 404, 64-70.

https://doi.org/10.1016/j.jmaa.2013.02.064

[39] Cheng, R. and Ross, W.T. (2015) Weak Parallelogram Laws on Banach Spaces and Applications to Prediction. Periodica Mathematica Hungarica, 71, 45-58. https://doi.org/10.1007/s10998-014-0078-4 